\title{
Distracción osteogénica del tercio medio facial. Reporte de dos casos clínicos
}

\section{Distraction osteogenesis of the midface. Two clinical cases report}

\section{Resumen}

La corrección quirúrgica de grandes asimetrías faciales son un desafío para el equipo quirúrgico. En dichos casos, los resultados de la corrección utilizando técnicas quirúrgicas convencionales son limitados, lo que hace necesario la utilización de otras herramientas terapéuticas. La distracción ósea (DO) es una de ellas, pues permite el estiramiento controlado del callo óseo previamente creado mediante osteotomías. La DO permite realizar cambios en el posicionamiento óseo de gran envergadura, favoreciendo el crecimiento óseo como mecanismo de acción. El objetivo de este artículo es describir los resultados quirúrgicos de DO intraoral del tercio medio facial utilizado en dos pacientes con severas asimetrías faciales asociadas a malformaciones de origen genético. Se relata el diagnóstico, la planificación, las herramientas tecnológicas utilizadas, técnica quirúrgica y los resultados obtenidos.

Palabras clave: Osteogénesis por distracción; Alargamiento óseo; Callo óseo; Osteotomía maxilar (fuente: DeCS BIREME).

\begin{abstract}
Surgical correction of major facial asymmetries is a challenge for the surgical team. In such cases, treatment results from conventional surgical techniques are limited, which requires using other therapeutic tools. Bone distraction is one of them, as it allows controlled stretching of the bone callus previously developed through osteotomies. Distraction osteogenesis allows making changes in large bone positioning, favoring bone growth as an action mechanism. This article aims to describe the surgical results of intraoral distraction osteogenesis of the midface used in two patients with severe facial asymmetries related to congenital malformations. Diagnosis, planning, technological tools, surgical techniques, and results obtained are explained.
\end{abstract}

Keywords: Distraction osteogenesis; Bone lengthening; Bony callus; Maxillary osteotomy (source: MeSH, NLM).

\section{Caso Clínico}

Coral Torres Manríquez 1,2,b , Carla Cittadini 1,b, Renato

Gunckel Muñoz 1,2,3,a , Marcelo Mardones Muñoz 1,2,3,a,

Rodrigo Bravo Ahumada 1,2,3,a

${ }^{1}$ Hospital San José, Servicio de Cirugía Oral y Máxilo-Facial, Santiago, Chile.

2 Universidad de Chile, Facultad Odontología, Santiago, Chile.

${ }^{3}$ Clínica Las Condes, Departamento de Cirugía Oral y

Máxilo-Facial, Santiago, Chile.

a Especialista en Cirugía y Traumatología Bucal y Máxilo-Facial.

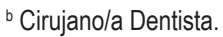

\section{Correspondencia:}

Rodrigo Bravo Ahumada: rbravo14@gmail.com San José 1196, Independencia, Región Metropolitana, Chile.

ORCID: 0000-0002-3480-1215

\section{Coautores:}

Coral Torres Manríquez: torresm.coral@gmail.com ORCID: 0000-0001-6637-6148

Carla Cittadini: odcarlacittadini@gmail.com ORCID: 0000-0002-1541-0665

Renato Gunckel Muñoz: renato.gunckel@gmail.com ORCID: 0000-0002-1443-1114

Marcelo Mardones Muñoz: drmardones@gmail.com ORCID: 0000-0003-4936-3294

\section{Editor:}

Juan Carlos Cuevas-González

Universidad Autónoma de Ciudad Juárez, México.

Conflicto de intereses: los autores declaran no tener conflictos de interés.

\section{Financiamiento: autofinanciado.}

\section{Recibido: 05/03/21}

Aceptado: 24/05/21

Publicado: 05/10/21 


\section{Introducción}

La distracción osteogénica (DO) en el territorio maxilofacial es una técnica quirúrgica que permite la corrección de grandes asimetrías faciales a través de la estimulación del callo óseo ${ }^{1}$.

Habitualmente este tipo de tratamientos es utilizado en malformaciones faciales severas, donde las técnicas convencionales, como la cirugía ortognática, por ejemplo, son limitadas en sus resultados ${ }^{2}$.

La DO es un tratamiento con múltiples beneficios ya que genera un crecimiento óseo progresivo estimulando también el tejido blando circundante, sin embargo, requiere de una cuidadosa evaluación del paciente y una correcta planificación preoperatoria, de manera de elegir el distractor más óptimo y definir los vectores de fuerza necesarios para lograr los resultados quirúrgicos deseados ${ }^{3}$.

Mientras que la literatura en su mayoría describe ampliamente el uso de DO para el tratamiento de las deformidades mandibulares, es menos conocido el tratamiento con DO del tercio medio facial.

En este estudio se presenta la técnica quirúrgica y los resultados de dos casos clínicos de pacientes diagnosticados con malformaciones congénitas asociadas a asimetrías faciales severas que afectan el tercio medio facial, que fueron tratadas con DO y distractores óseos intraorales.

\section{Reporte de casos}

\section{Caso clínico 1}

Paciente sexo masculino 15 años de edad, portador de síndrome de Warkany (trisomía 8) asociado a dismorfismo craneofacial e insuficiencia renal tratada con trasplante renal.

Presenta biotipo braquifacial severo con clase III esqueletal por falta de desarrollo de tercio medio facial y fisura labio palatina derecha operada, generando una alteración oclusal con mordida invertida y discrepancia intermaxilar de $10 \mathrm{~mm}$, asociado a una función respiratoria y masticatoria alterada.

El paciente registra un historial previo de hace 3 años de tratamiento de ortodoncia y cirugía de DO alveolar con distractor tipo Liou. No obstante, debido a la persistencia de la deformidad facial, requirió de una nueva planificación de cirugía de DO del tercio medio facial.

Para la planificación pre quirúrgica consideramos la planificación virtual tridimensional (PVT), herramienta tecnológica realizada con de un software de planificación, y el uso de modelos estereolitográficos del esqueleto facial del paciente, para simular el diseño de la osteotomía en los maxilares, los movimientos esqueléticos requeridos, la selección del distractor óseo apropiado y su localización tridimensional.

De acuerdo a la PVT se planificó un avance del hueso maxilar de $22 \mathrm{~mm}$ considerando el avance necesario y un rango de pérdida en el avance aproximado de un 30\% según describe la literatura, seleccionando para ello dos distractores internos de tipo Maxilar Telescópico con un rango de distracción de 30 mm (KLS Martin Group, Tuttlingen, Alemania) ${ }^{4,5}$. Se simuló la localización tridimensional de los distractores en el modelo estereolitográfico, la osteotomía planificada, la paralelización de los distractores y la activación de ellos para chequear su vector de fuerza, correcta función y localización (Figura 1).

Bajo anestesia general con intubación nasotraqueal, se realizó una incisión lineal en fondo de vestíbulo superior, decolado y exposición de pared anterior y tuberosidad del hueso maxilar y cuerpo de hueso cigomático, con posterior osteotomía tipo LeFort I. Se realizó la fractura quirúrgica y se chequeó la movilidad del hueso maxilar. Se instalaron los distractores de tipo Maxilar Telescópico según planificación, los que fueron activados intra operatoriamente para comprobar la movilidad del hueso maxilar. Se suturaron los tejidos blandos por planos (Figura 2).

El paciente fue hospitalizado el mismo día de la cirugía, se indicó terapia antibiótica endovenosa de amoxicilina con ácido clavulánico. El tratamiento farmacológico se complementa con antibioticoterapia vía oral por 7 días, y para el manejo del dolor se indica paracetamol y keteprofeno. Se indico alimentación con dieta blanda por 30 días posterior a la activación.

El paciente evolucionó favorablemente en el post operatorio inmediato, 5 días posterior a la cirugía se inició el periodo de activación de los distractores con $0,5 \mathrm{~mm}$ cada 12 horas, para ganancia de $1 \mathrm{~mm}$ diario. Transcurridos 60 días con los distractores óseos instalados, el hueso maxilar logró una distracción final de $22 \mathrm{~mm}$. Se decidió retirar los distractores en forma precoz, sin esperar una consolidación ósea, debido a la incomodidad presentada por el paciente (Figuras 3 y 4 ).

Durante la segunda intervención quirúrgica, se evidenció el avance maxilar logrado por la distracción osteogénica y se reemplazaron los distractores por placas de osteosíntesis para favorecer la fijación maxilar. Se lograron resultados de armonía facial aceptable, oclusión estable y funcional, continuando con el tratamiento ortodóncico postoperatorio.

\section{Caso clínico 2}

Paciente sexo masculino 26 ańos de edad, portador de deleción de cromosoma 6 asociado a dismorfosis craneofacial.

Presenta biotipo dólicofacial con clase III esqueletal asociado a hipoplasia maxilomandibular, generando alteración oclusal con mordida invertida y discrepancia intermaxilar de $15 \mathrm{~mm}$, asociado a una función masticatoria, respiratoria alterada y presentaba antecedentes previos de cirugía de disyunción maxilar de hace 1 año, previo a la cirugía de distracción maxilar. Debido a su deformidad facial, requiere de planificación de cirugía de DO del tercio medio facial.

Para la planificación pre quirúrgica consideramos la planificación virtual tridimensional (PVT), herramien- 

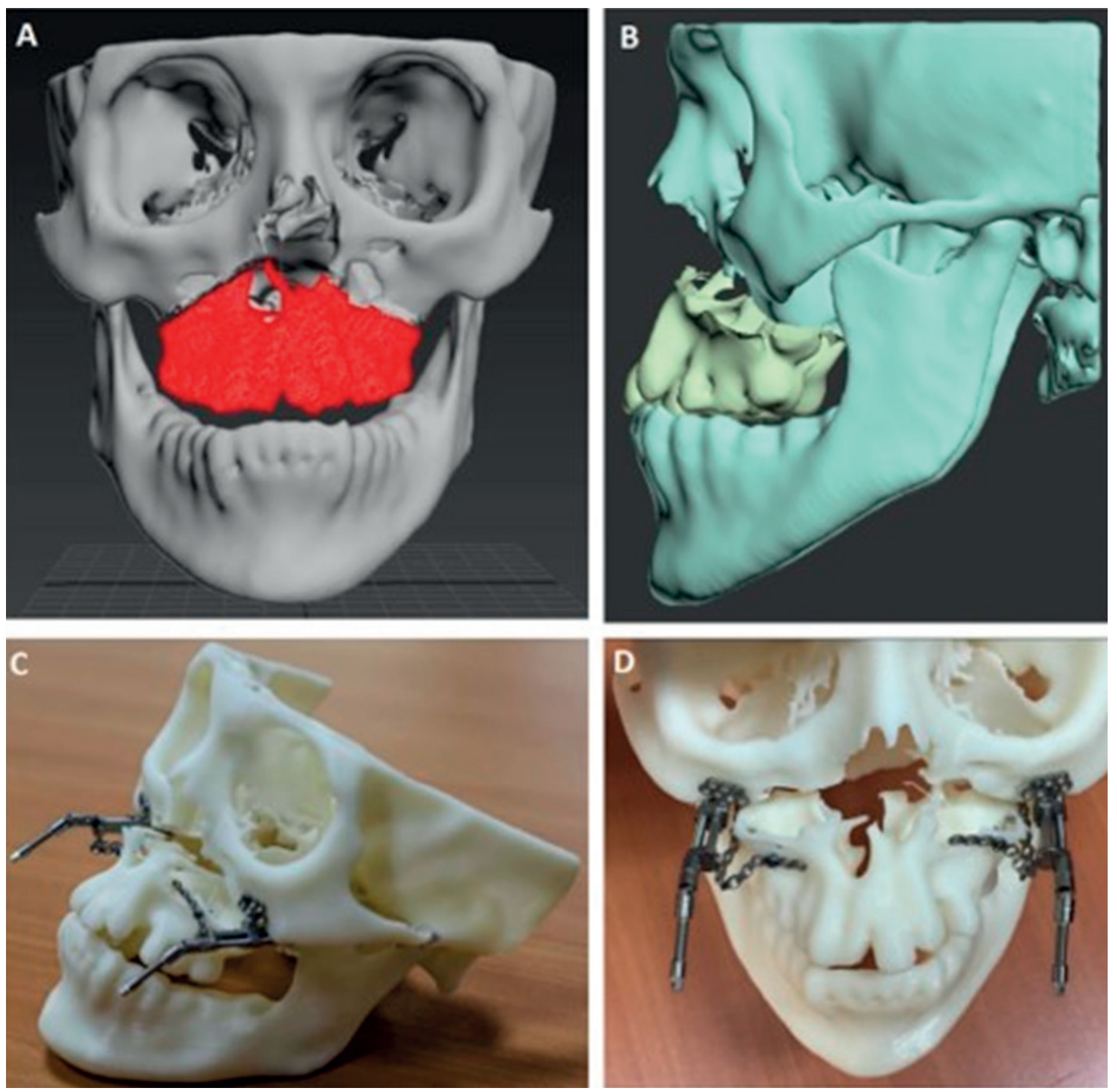

Figura 1. Planificación virtual tridimensional de caso clínico 1. A y B. Simulación de osteotomía en software de planificación. C y D. Localización de distractores en modelo estereolitográfico
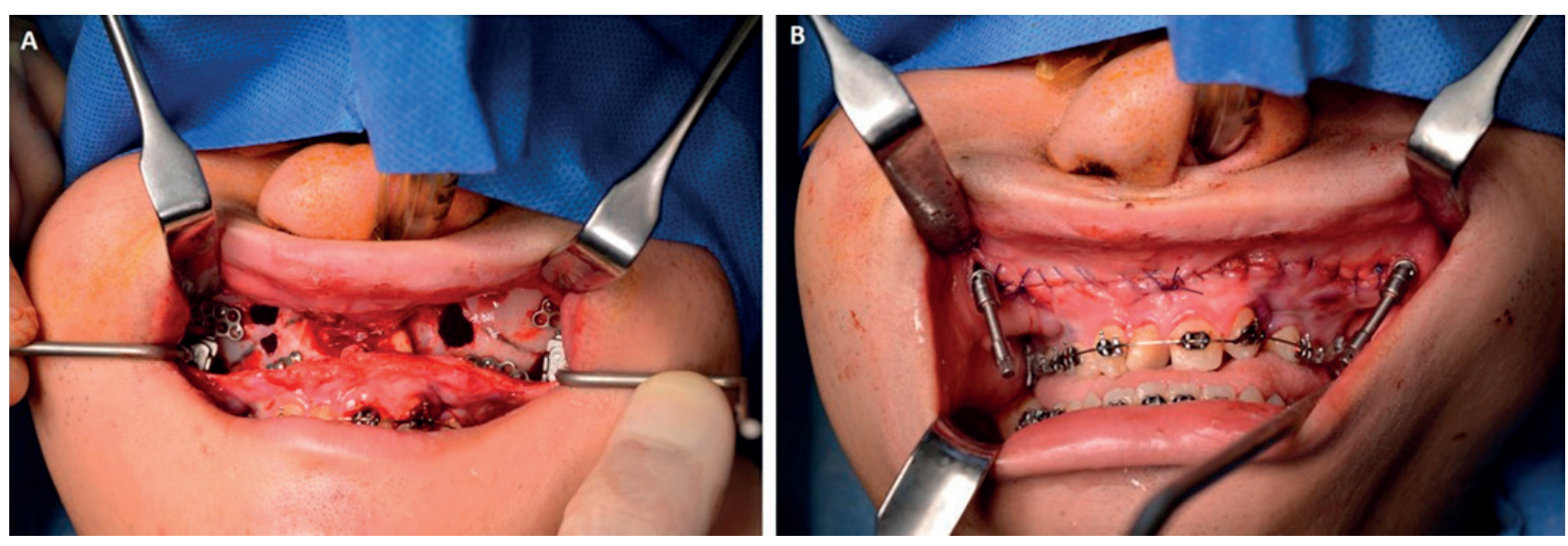

Figura 2. Cirugía de instalación de distractores óseos de caso clínico 1. A. Instalación de distractores. B. Sutura de tejido blando 

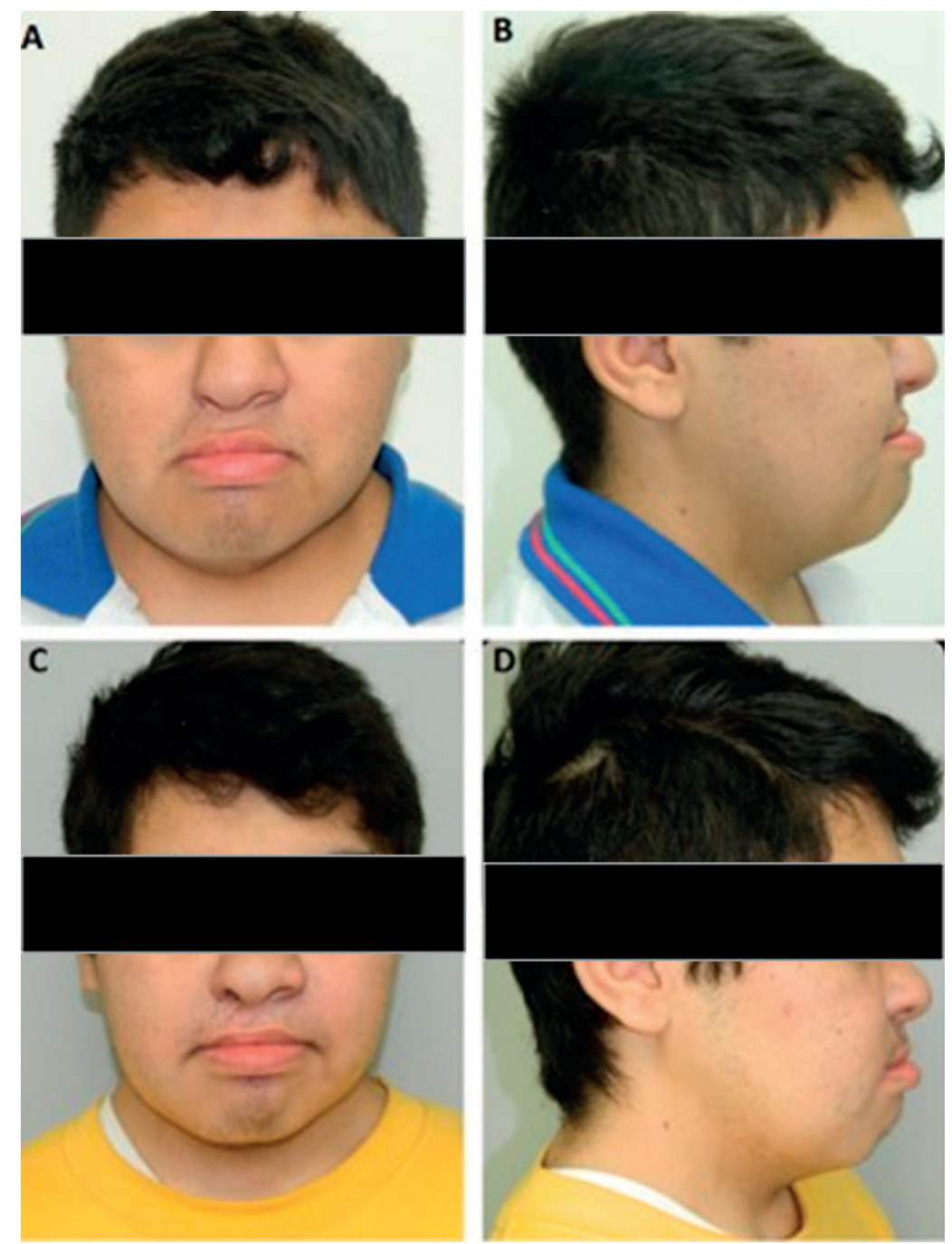

Figura 3. Imágenes extraorales pre y post quirúrgica de caso clínico 1. A y B. Plano frontal y sagital pre quirúrgica. C y D. Plano frontal y sagital post quirúrgica
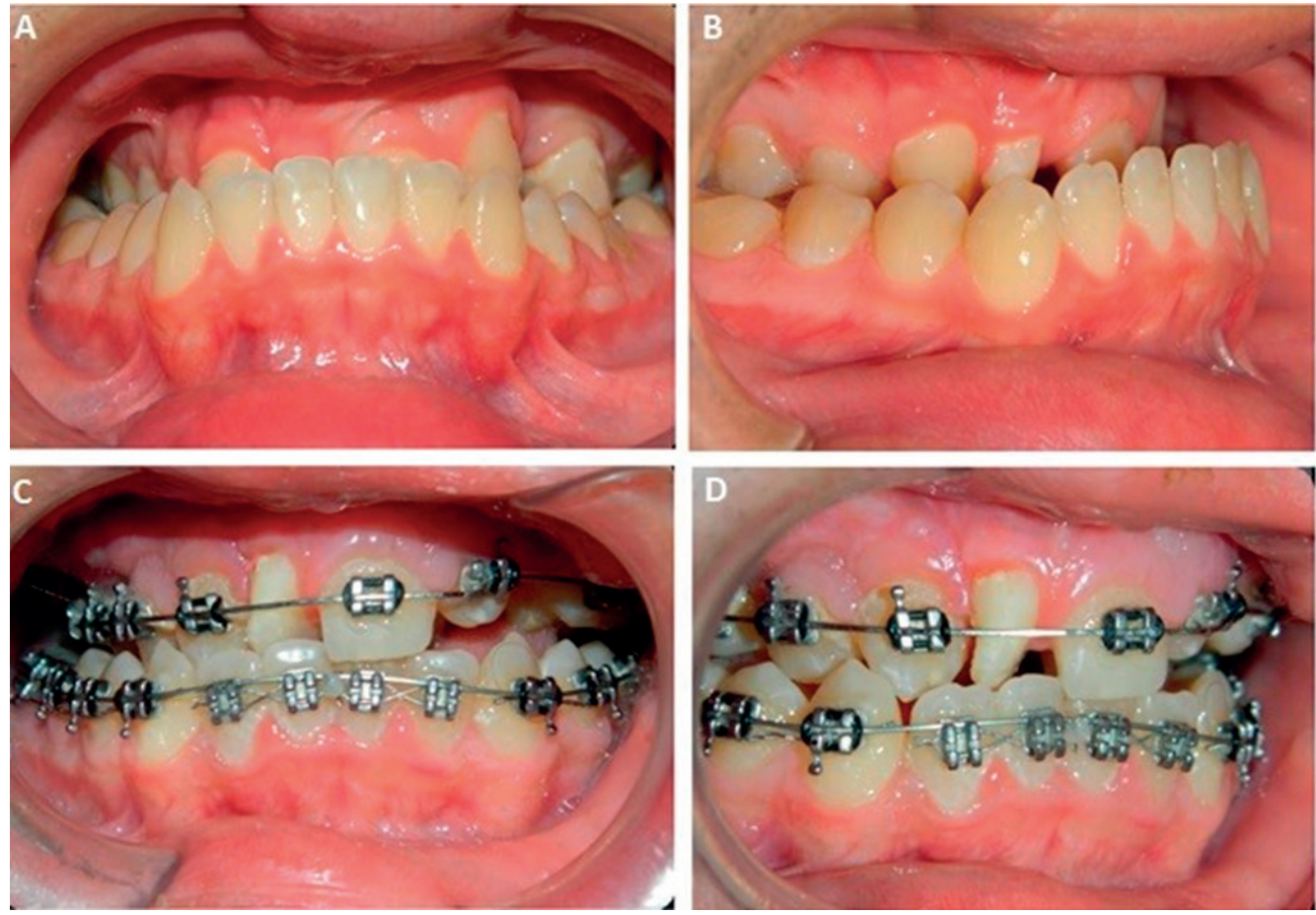

Figura 4. Imágenes intraorales pre y post quirúrgica de caso clínico 1. A y B. Oclusión pre quirúrgica. C y D. Oclusión post quirúrgica 
ta tecnológica realizada por medio de un software de planificación, y el uso de modelos estereolitográficos del esqueleto facial del paciente, para simular el diseño de la osteotomía en los maxilares, los movimientos esqueléticos necesarios, la selección del distractor óseo apropiado y su localización tridimensional.

De acuerdo a la PVT se requirió un avance del hueso maxilar de $28 \mathrm{~mm}$, seleccionando para ello dos distractores internos de tipo Maxilar Telescópico con un rango de distracción de $30 \mathrm{~mm}$ (KLS Martin Group, Tuttlingen, Alemania), considerando el avance necesario y una sobre corrección suponiendo una pérdida aproximada del 30\% del avance maxilar ${ }^{4,5}$. Se simuló la localización tridimensional de los distractores en el modelo estereolitográfico, la osteotomía planificada, la paralelización de los distractores y la activación de ellos para chequear su vector de fuerza, correcta función y localización (Figura 5).

Bajo anestesia general con intubación nasotraqueal, se realizó una incisión lineal en fondo de vestíbulo superior, decolado y exposición de pared anterior y tuberosidad del hueso maxilar y cuerpo de hueso cigomático, con posterior osteotomía tipo LeFort I. Se realizó la fractura quirúrgica y se chequeó la movilidad del hueso maxilar. Se instalaron los distractores de tipo Maxilar Telescópico según planificación, los que fueron activados intra operatoriamente para comprobar la movilidad del hueso maxilar. Se suturaron los tejidos blandos por planos. (Figura 6)

El paciente fue hospitalizado el mismo día de la cirugía, se indicó terapia antibiótica endovenosa de amoxicilina con ácido clavulánico. El tratamiento farmacológico se complementa con antibioticoterapia vía oral por 7 días, y para el manejo del dolor se indica paracetamol y keteprofeno. Se indico alimentación con dieta blanda por 30 días posterior a la activación.

El paciente evolucionó favorablemente en el post operatorio inmediato, 5 días posterior a la cirugía se inició el periodo de activación de distractores con $1 \mathrm{~mm}$ diario. Una semana después, se activaron un poco más para una ganancia ósea de 1,2 $\mathrm{mm}$ diarios. Transcurridos 36 días desde la cirugía de instalación de los distractores óseos, se decidió retirarlos de forma prematura sin esperar una consolidación ósea, debido a molestias manifestadas por el paciente (Figuras 7 y 8 ).

En esta segunda cirugía se observó el avance maxilar logrado. Los distractores fueron reemplazados por placas
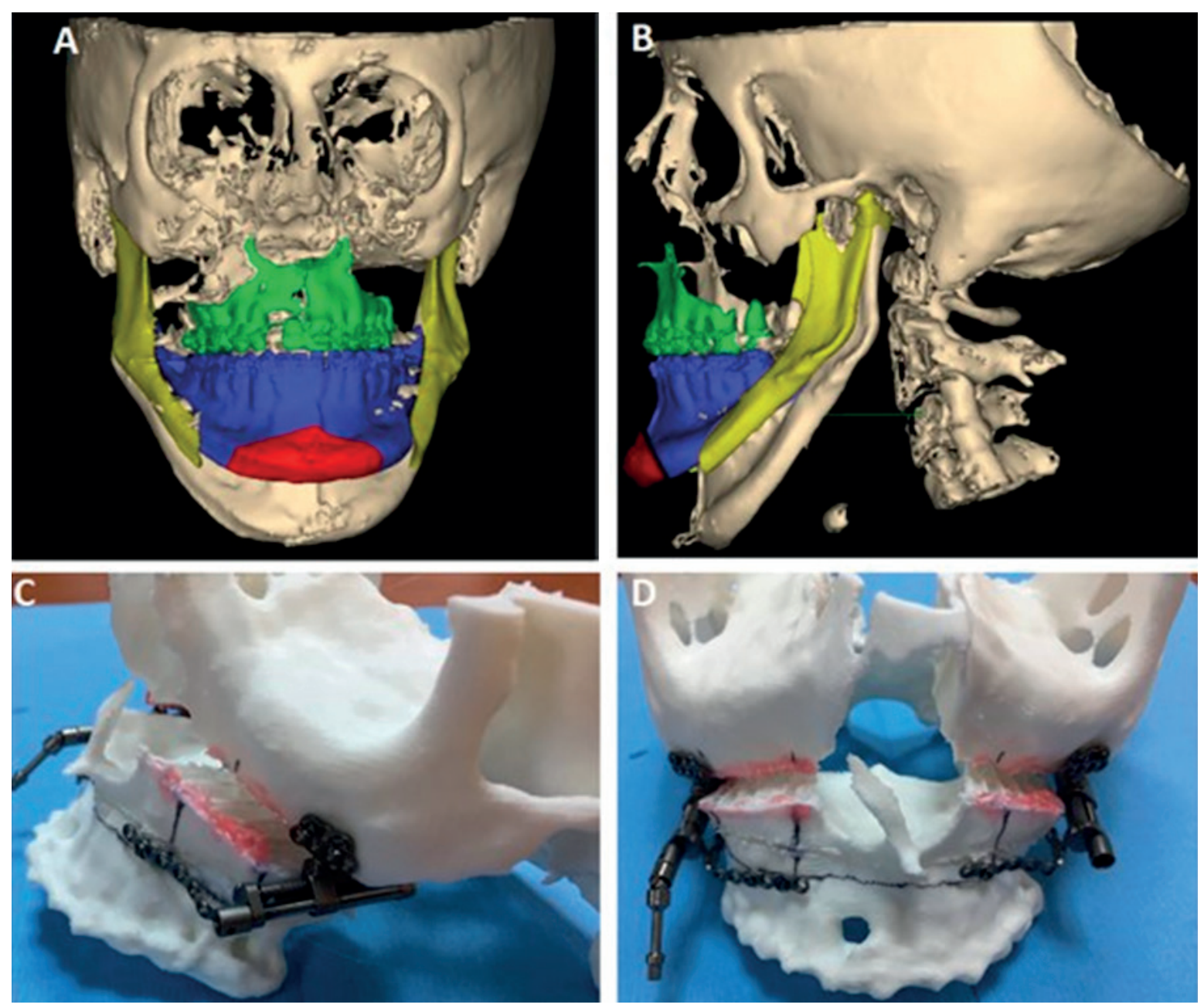

Figura 5. Planificación virtual tridimensional de caso clínico 2. A y B. Simulación de osteotomía en software de planificación. C y D. Localización de distractores en modelo estereolitográfico 

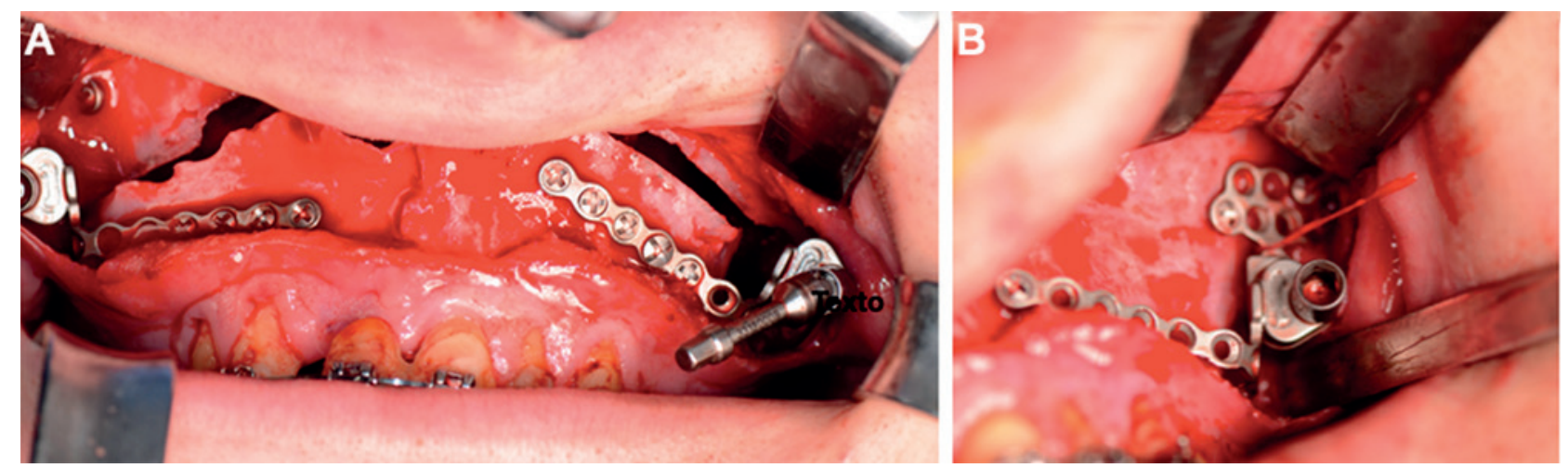

Figura 6. Cirugía de instalación de distractores óseos de caso clínico 2. A. Instalación de distractores. B. Distractor óseo lado izquierdo
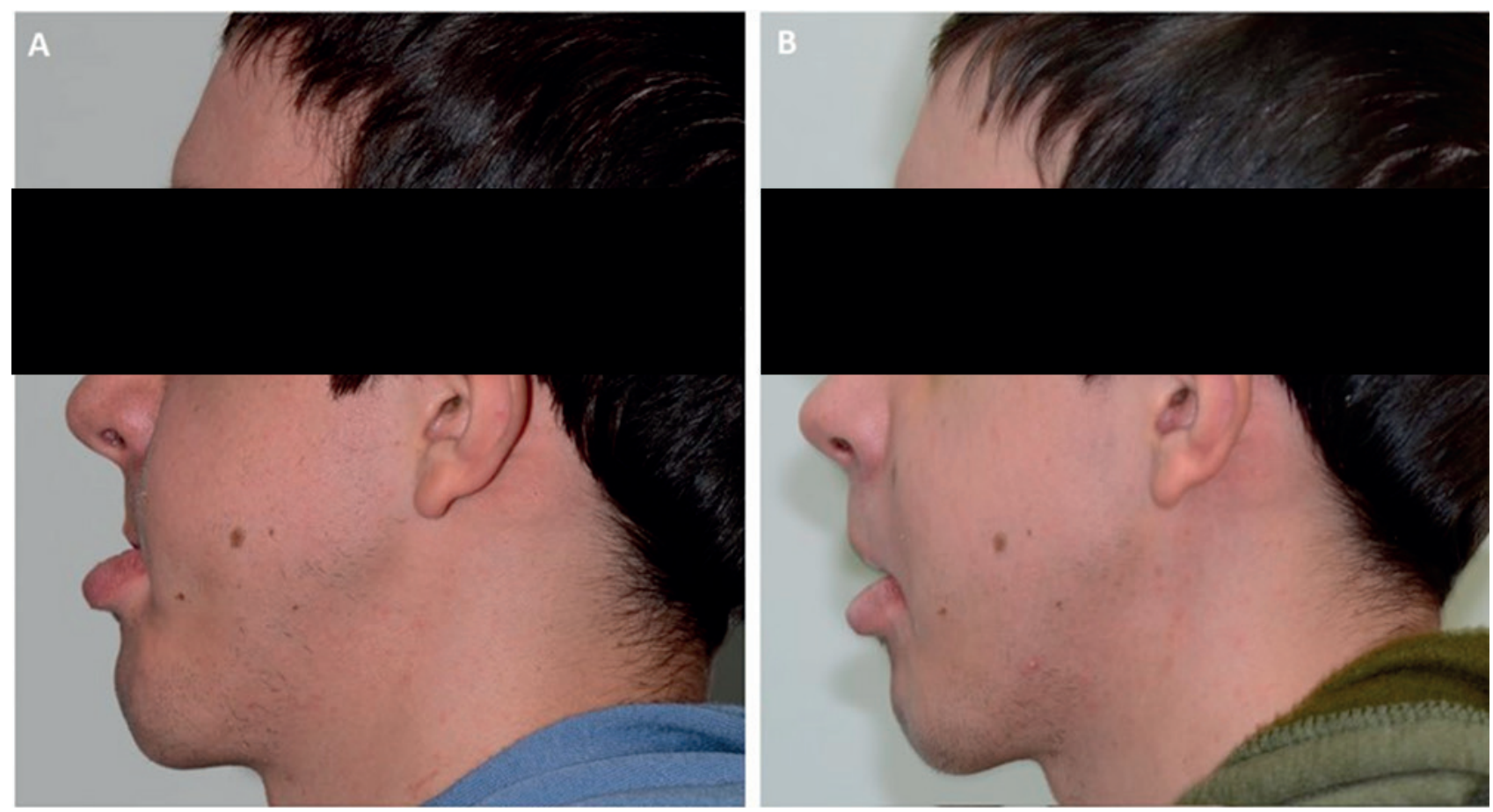

Figura 7. Imágenes extraorales de caso clínico 2. A. Pre quirúrgico. B. Post quirúrgico
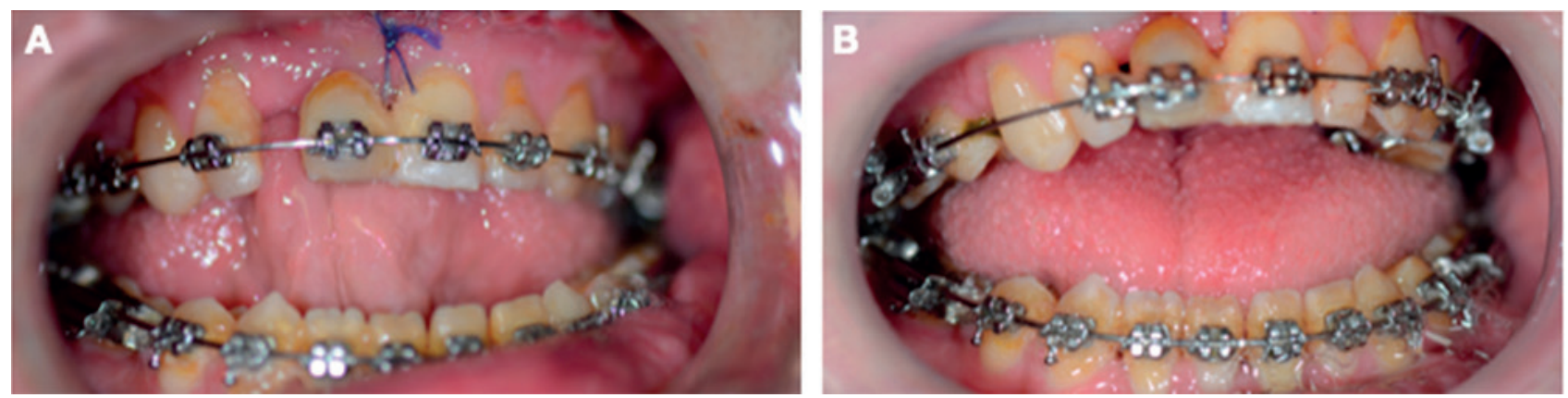

Figura 8. Imágenes post quirúrgica de caso clínico 2. A. Imagen control posterior instalación distractores. B. Imagen control retiro de distractores 
de osteosíntesis para favorecer la fijación maxilar. Posteriormente el paciente requirió una cirugía ortognática para reposicionar la mandíbula, recuperar la función oclusal y respiratoria.

\section{Discusión}

Presentamos dos casos clínicos con malformaciones faciales de origen genético con una gran hipoplasia del tercio medio facial y acentuada discrepancia intermaxilar, que fueron tratadas con DO. La DO se describe como la estimulación del callo óseo previamente osteotomizado, que separa gradualmente los segmentos óseos entre sí por la tracción ejercida por el distractor ${ }^{4}$. Este tipo de tratamiento fue elegido en estos pacientes por sobre la cirugía ortognática convencional debido a la gran discrepancia entre las bases esqueletales y la necesidad de avance del maxilar superior a $10 \mathrm{~mm}$ en cada uno de los pacientes ${ }^{6}$. La cirugía ortognática logra buenos resultados en discrepancias intermaxilares entre $4-9 \mathrm{~mm}$, pero sus resultados son menos predecibles en movimientos de mayor magnitud. Múltiples estudios han demostrado que un avance $>10 \mathrm{~mm}$, especialmente en pacientes con antecedentes de fisura labio palatina, presentan una alta tasa de recidiva del $20 \%$ al $60 \%{ }^{2}$. La discrepancia intermaxilar en los casos presentados era superior a 10 $\mathrm{mm}$, y estaban asociados a malformaciones faciales de origen genético, motivo por el que se decidió realizar una cirugía de DO. Esto coincide con Wang et al. ${ }^{7}$ que indica que la DO maxilar total proporciona un mayor avance maxilar y es preferible ante la cirugía ortognática en pacientes jóvenes con malformaciones faciales.

El proceso de DO comienza con una cuidadosa evaluación y planificación preoperatoria. Durante la planificación preoperatoria consideramos la PVT, tecnología que aporta mayor información tridimensional ${ }^{8}$. Optamos por este tipo de planificación debido a que determina el posicionamiento tridimensional con precisión de los distractores en el maxilar, simula los movimientos esqueletales requeridos, diseño de la osteotomía y determina cuánto avance de tejido óseo se generará, ventaja que no puede garantizarse mediante el uso de cirugía de modelos clásica o cefalometría bidimensional ${ }^{9}$. Para su realización, trabajamos con modelos estereolitográficos de cada paciente (modelo tridimensional del esqueleto facial) y un software de planificación para realizar las osteotomías virtuales y simular el movimiento de los segmentos óseos. Esta simulación ayudó en la selección del distractor apropiado, reduciendo así el tiempo quirúrgico y las complicaciones intraoperatorias ${ }^{10,11}$.

Durante la planificación también se consideró una sobre corrección en la distracción de aproximadamente un $30 \%$, apoyándose en lo que describe la literatura en cuanto a pérdida de avance que existe entre los segmentos óseos distraídos, principalmente asociado a cirugías previas, bridas cicatriciales, estímulo muscular y de otros tejidos blandos sobre los segmentos óseos ${ }^{3,4}$.

La planificación previa con modelo esterolitográfico también permitió dar un correcto paralelismo entre los distractores, de manera de no generar fuerzas convergentes o divergentes que impidieran la correcta activación de los mismos y permitió observar además cómo se comportaría el segmento maxilar durante la distracción ${ }^{12}$.

El mercado ofrece distractores extraorales e intraorales, cada uno con ventajas y desventajas ${ }^{3}$. En estos casos se optó por distractores intraorales por dos razones. La principal fue el factor psicológico de los pacientes los cuáles no deseaban portar un dispositivo en su cara que fuera visible. Otro factor que incidió también en la elección fue que solamente se requería un movimiento unidireccional de avance del tercio medio facial.

En nuestros pacientes observamos un buen comportamiento de los distractores en relación a los vectores de fuerza planificados, sin embargo, las molestias de portar un aparato dentro de la boca, que generaba úlceras importantes en la mejilla, fue un factor negativo y que influyó también en el retiro de los distractores en forma precoz, siendo imposible esperar el tiempo de consolidación ósea requerida.

Como una forma de prevenir la pérdida del avance maxilar logrado y favorecer la cicatrización ósea, se planificó el retiro de los distractores y su reemplazo por placas de osteosíntesis, de manera que aseguraran la estabilidad del maxilar. Aun así, se pudo visualizar efectivamente la pérdida de avance en el post operatorio tardío, sin embargo, el objetivo de lograr una oclusión y una relación intermaxilar más funcional se consiguió y mejoró el aspecto facial que era una de las expectativas de los pacientes, quienes indicaron estar conformes con su tratamiento.

La DO en el territorio maxilofacial es un tratamiento desafiante y un protocolo exitoso depende de múltiples factores, incluyendo un diagnóstico y planificación precisos, una técnica quirúrgica cuidadosa, las expectativas y el cumplimiento del paciente y sus factores psicológicos. La planificación virtual tridimensional se convierte en el Gold Standard actual que permite una predicción precisa del comportamiento de las estructuras óseas.

El distractor ideal actualmente no existe y no están libres de morbilidad en los pacientes, en nuestro caso el principal inconveniente fue la incomodidad del distractor en la cavidad oral que indicó el retiro precoz de ellos, una vez terminada la distracción requerida.

\section{Referencias bibliográficas}

1. Austin SL, Mattick CR, Waterhouse PJ. Distraction osteogenesis versus orthognathic surgery for the treatment of maxillary hypoplasia in cleft lip and palate patients: a systematic review. Orthod Craniofac Res. 2015;18(2):96108. DOI:10.1111/ocr.12063.

2. Burstein F, Soldanska M, Granger M, Berhane C, Schoemann M. Initial Experience With a New Intraoral Midface Distraction Device. J Craniofac Surg. 2015;26(4):12241228. DOI:10.1097/SCS.0000000000001728.

3. Meling TR, Høgevold HE, Due-Tønnessen BJ, Skjelbred P. Midface distraction osteogenesis: internal vs. external devices. Int J Oral Maxillofac Surg. 2011;40(2):139145. DOI:10.1016/j.ijom.2010.10.009. 
4. Balaji SM, Balaji P. Comparison of Midface Advancement by External and Internal Craniofacial Distraction Osteogenesis. Ann Maxillofac Surg. 2018;8(2):200-205. DOI:10.4103/ams.ams_234_18.

5. Brochures, catalogs and flyers [Internet]. Klsmartin. com. [citado el 8 de septiembre de 2021]. Disponible en: https://www.klsmartin.com/en/media-library/brochures-catalogs-flyers/

6. Losa P, Burgueño M, Gómez E. Distracción maxilar con dispositivos internos en pacientes fisurados: Planificación virtual y transferencia de datos al campo quirúrgico. Rev. Esp Cirug Oral y Maxilofac. 2017;39(2):80-84. DOI: 10.1016/j.maxilo.2016.05.005.

7. Wang Y, Li J, Xu Y, Huang N, Shi B, Li J. Accuracy of virtual surgical planning-assisted management for maxillary hypoplasia in adult patients with cleft lip and palate. J Plast Reconstr Aesthet Surg. 2020;73(1):134-140. DOI:10.1016/j.bjps.2019.07.003.

8. Robiony M, Salvo I, Costa F, et al. Virtual reality surgical planning for maxillofacial distraction osteogenesis: the role of reverse engineering rapid prototyping and cooperative work. J Oral Maxillofac Surg. 2007;65(6):11981208. DOI:10.1016/j.joms.2005.12.080.
9. Scolozzi P, Herzog G. Computer-Assisted Virtual Planning for Surgical Guide Manufacturing and Internal Distractor Adaptation in the Management of Midface Hypoplasia in Cleft Patients. Cleft Palate Craniofac J. 2017;54(4):457-464. DOI:10.1597/15-208.

10. Gateno J, Teichgraeber JF, Xia JJ. Three-dimensional surgical planning for maxillary and midface distraction osteogenesis. J Craniofac Surg. 2003;14(6):833-839. DOI:10.1097/00001665-200311000-00004.

11. Rubio-Palau J, Prieto-Gundin A, Cazalla AA, et al. Three-dimensional planning in craniomaxillofacial surgery. Ann Maxillofac Surg. 2016;6(2):281-286. DOI:10.4103/2231-0746.200322.

12. Sándor GK, Ylikontiola LP, Serlo W, Carmichael RP, Nish IA, Daskalogiannakis J. Distraction osteogenesis of the midface. Oral Maxillofac Surg Clin North Am. 2005;17(4):485-501. DOI:10.1016/j. coms.2005.06.005. 